\title{
Dynamic modelling for thermal micro-actuators using thermal networks
}

\author{
Beatriz López-Walle ${ }^{\mathrm{a}, 1}$, Michaël Gauthier*,a ${ }^{*}$ Nicolas Chaillet $^{\mathrm{a}}$ \\ ${ }^{a}$ FEMTO-ST Institute, CNRS/UFC/ENSMM/UTBM, Automatic Control and \\ MicroMechatronic Systems Department (AS2M), 24 rue Alain Savary, 25000 Besançon, \\ France
}

\section{Abstract}

Thermal actuators are extensively used in microelectromechanical systems (MEMS). Heat transfer through and around these microstructures are very complex. Knowing and controlling them in order to improve the performance of the micro-actuator, is currently a great challenge. This paper deals with this topic and proposes a dynamic thermal modelling of thermal micro-actuators. Thermal problems may be modelled using electrical analogy. However, current equivalent electrical models (thermal networks) are generally obtained considering only heat transfers through the thickness of structures having considerable height and length in relation to width (walls). These models cannot be directly applied to micro-actuators. In fact, microactuator configurations are based on 3D beam structures, and heat transfers occur through and around length. New dynamic and static thermal networks

\footnotetext{
${ }^{*}$ Corresponding author. Tel.:+33(0)381 402 810; fax: +33 (0)381 402809.

Email addresses: beatriz.lopezwl@uanl.edu.mx (Beatriz López-Walle), michael.gauthier@femto-st.fr (Michaël Gauthier), nicolas.chaillet@femto-st.fr (Nicolas Chaillet)

${ }^{1}$ Current address: CIIDIT - Universidad Autónoma de Nuevo León, PIIT Monterrey, C.P. 66600, Nuevo León, Mexico.
} 
are then proposed in this paper. The validities of both types of thermal networks have been studied. They are successfully validated by comparison with finite elements simulation and analytical calculations.

Key words:

Micro-actuators, Thermal modelling, Electrical analogy, Thermal network

\section{Nomenclature}

The notations used in this article are summarized in table 1.

\section{Introduction}

4 The thermal actuation is widely present in active microsystems, either by 5 inherent design or combining two or more energy domains such as optical, 6 mechanical or electrical $[1,2,3]$.

7 One of the earliest (1970's) and most commercially successful application 8 of thermal microelectromechanical systems (MEMS) is the ink-jet printer 9 head [4]. Recent applications including microlens [5, 6], microprobes [7, 8],

$$
\begin{aligned}
& \text { microsensors }[9,10,11,12,13] \text { and micro-actuators, are rapidly gaining im- } \\
& \text { portance too. For example, a thermal microlens tunes its focal lens by tem- } \\
& \text { perature, which is easily controlled via managing the current input of the } \\
& \text { heater }[6] \text {. An on-wall in-tube flexible thermal sensor is able to measure the } \\
& \text { flow rate under both developing and fully developed flow conditions. The } \\
& \text { resistance of the sensor linearly changes with temperature [13]. } \\
& \text { Thermal micro-actuators are a very popular actuation technology in MEMS. } \\
& \text { They commonly exploit differential thermomechanical expansion of materi- } \\
& \text { als, known as the thermomechanical effect, resulting generally from Joule }
\end{aligned}
$$


Table 1: Nomenclature

\begin{tabular}{llrlll}
\hline$a$ & height & $\mathrm{m}$ & $T$ & temperature & $\mathrm{K}$ \\
$b$ & width & $\mathrm{m}$ & $V$ & voltage & $\mathrm{V}$ \\
$C_{p}$ & specific heat (constant pressure) & $\mathrm{J} / \mathrm{kg} \mathrm{K}$ & $x, y, z$ & Cartesian coordinates & $\mathrm{m}$ \\
$C_{t h 0}$ & thermal capacitor & $\mathrm{J} / \mathrm{K}$ & $Z$ & thermal impedance & \\
$d$ & characteristic size & $\mathrm{m}$ & Greek & symbols \\
$h$ & heat transfer coefficient & $\mathrm{W} / \mathrm{m}^{2} \mathrm{~K}$ & $\delta$ & static validity criterion & \\
$i$ & current & $\mathrm{A}$ & $\nu$ & dynamic validity criterion & $\mathrm{rad} / \mathrm{s}$ \\
$k$ & thermal conductivity & $\mathrm{W} / \mathrm{m} \mathrm{K}$ & $\omega$ & angular frequency & $\mathrm{W}$ \\
$l$ & length & & $\phi$ & heat Laplace transform & $\mathrm{kg} / \mathrm{m}^{3}$ \\
$P$ & lateral perimeter & $\mathrm{m}$ & $\rho$ & mass density & $\mathrm{K}$ \\
$Q_{h}$ & heat convection & $\mathrm{W}$ & $\theta$ & temperature Laplace transform & \\
$Q$ & heat flux & $\mathrm{W}$ & $S u b s c r i p t s$ & \\
$R_{c 0}$ & conduction thermal resistance & $\mathrm{K} / \mathrm{W}$ & 1 & lateral face, at $x=0$ & \\
$R_{v 0}$ & convection thermal resistance & $\mathrm{K} / \mathrm{W}$ & 2 & lateral face, at $x=l$ & \\
$S$ & lateral surface & $\mathrm{m}$ & ext & external & \\
$t$ & time & $\mathrm{s}$ & lin & linear & \\
& & & st & static & \\
& & &
\end{tabular}

heating. The common actuation geometries of this kind of micro-actuators are multimorph $[14,15,16]$, U-shape $[1,17,18,19,20,21]$ and V-shape $[17,22,23,24,25]$. In all of them, the prevalent geometry is the beam. Properties of thermal micro-actuators strongly depend on their geome- 
try structure and material properties, as well as the applied current. It is therefore important to model the thermal micro-actuator in order to improve or optimize its design. Thermal micro-actuators are used in microgrippers [16, 19, 25, 26, 27], microheaters [28, 29], micromotors [30], microrobots [31], micromirrors [14], ice gripping [32], etc. Direct applications of these devices are electrics, optics, electronics, mechanics and biomedicine [30, 33]. Thermal actuators are usually rather slow, due to thermal time constants typically in the upper millisecond range [34]. In smaller structures, however, it has been shown that substantially higher speeds can be attained, because thermal time constants scale linearly with decreasing surface [30, 35]. Compared to their counterparts such as electrostatic or piezoelectric actuators, thermal actuation provides larger forces [36]: for typical configurations, thermal actuation provides 4 orders of magnitude higher energy density than electrostatic actuation $\left(450 \mu \mathrm{N} . \mathrm{mm}^{-2}\right.$ for thermal actuation, and $20 \mu \mathrm{N} . \mathrm{mm}^{-2}$ for electrostatic actuation [22]), and 1 to 2 orders of magnitude higher energy density than piezoelectric actuation $[1,30]$. Thermal micro-actuators have a high reliability and are also easier to control, compared to shape memory alloy actuators. In addition, they are usually simpler to be fabricated, contrary to magnetic actuators, for instance, that may require special materials in the fabrication process [22]. Some of the thermal micro-actuators have been made on silicon, polysilicon, and nickel structural components. Metalbased electrothermal micro-actuators provide a larger output displacement with a smaller input voltage. However, they generally suffer from mechanical deficiencies, such as fatigue and aging [33].

Fabrication techniques of thermal micro-actuators are well-known mi- 
crotechnologies such as bulk micromachining, wet and dry etching processes, surface micromachining and LIGA processes, including laser micromachining $[1,2,6,14,24,37]$. Managing thermal phenomena in thermal microactuators is a key factor for future progress in their optimization. Modelling heat transfers through the actuator and its surroundings are fundamental for understanding, predicting and controlling the temperature distribution, and consequently the device response characteristics. This paper deals with this problem and proposes a dynamic thermal analysis using electrical analogy. This last is generally used to model heat transfers occurring through the thickness of structures with geometries as walls, where height and length are considerable in relation to width. Here, we develop equivalent electrical models of long structures, which are currently found in thermal micro-actuators. In these thermal structures, heat transfers are present through and around the length.

Being the analysis presented in this paper useful for the MEMS community dealing with thermal problems, in Section 3 we present different approaches to model these problems, particularly in micro-electronics and thermal micro-actuators. The beam geometry, currently found in thermal micro-actuators, is analysed dynamically in Section 4 via electrical analogy. In Section 5 we determine the validity of the proposed equivalent electrical models, and they are validated by comparing them with finite elements simulations and analytical calculations in Section 6. 


\section{Thermal modelling in microdevices}

Thermal effects have an obvious impact in the performance and reliability of electronic systems and thermal devices [38]. Efforts to model the electrothermal actuators and thermal effects in electronic systems have been focused on analytical modelling, finite-element methods, lumped parameters based on electrical analogy, and model order reduction. Several examples of these techniques are presented in this section.

\subsection{Analytical modelling}

In practice, an analytical solution provide very accurate models, and it may easily consider constraints. Liao et al. theoretically modelled an electrothermal micro-actuator for bidirectional motion to forecast the relationship between applied voltage and displacement [1]. A one-dimensional conductive heat transfer analysis of two types of thermal actuators (U-shaped and V-shaped) are presented by Hickey et al. [17]. Thermal time constants were predicted using this model. Robert et al. predicted the shape of a thermal and electrostatic micro-actuator versus the temperature [3]. An algorithm has been also developed in order to evaluate the damping behavior of a microswitch taking the micro-actuator deflection into account. Li and Uttamchandani analysed a modified asymmetric micro-electrothermal actuator [18]. The aim of this analysis was to calculate the optimum dimensions of the hot arm of the actuator to maximize the deflection of the actuator before the onset of thermal failure. A theoretical model of the actuation stress of a polymeric (SU-8) thermal micro-actuator with embedded silicon microstructure has been realized by Lau et al. [39]. The analytical model 
of Boutchich et al. predicted the dependence of the restoring force on the input electrical power and topology of a thermal actuator [15]. A two-step analytical model of a four-hot arm U-shape electrothermal actuator that can achieve bidirectional motion in two axes has been developed by Elbuken et al. [40]. Finally, Mayyas and Stephanou obtained closed-form solutions for the thermal modelling of a general 5 non-homogeneous, lineshape microbeam's actuator using 1-D steady state heat equations under both heat conduction and convection [41].

Finite element (FE) based simulations $[1,17,40,41]$ or experimental measurements $[3,15,17,18]$ were usually performed to validate analytical models. Analytic solutions for describing thermal problems are only available for simple geometries. For complex geometries, either numerical methods or approximations may be used.

\subsection{Finite element modelling}

Electrothermal devices are traditionally simulated with finite element method (FEM) due to the complex coupling of the electrical, thermal and mechanical problem [42]. FEM analysis is principally used to demonstrate the feasibility of the design and to simulate the behaviour; for example, the relationship between the applied voltage and the displacement [1, 37, 43, 44], or the effects of geometrical and material stiffness variations on the performance [45].

The principal drawbacks of implementing analytical and FEM analysis in electrothermal microdevices are regarded to the long simulation time, and the time required to build-up the model in the FEM software. Faster methods become necessary. 


\subsection{Modelling using electrical analogy}

Electrical analogy has been used for the first time by Paschkis and Baker in 1942 to solve the unsteady-state and unidirectional heat conduction equation in a plate [46]. Nevertheless, the principles of the electrical analogy applied to unsteady-state heat conduction problems have been proposed by Jakob in 1949 [47]. Ever since, equivalent electrical models, so-called thermal networks, seem a relatively simple, but sufficiently accurate and powerful tool for simulating real thermal systems and microsystems [48, 49, 50, 51].

Dynamic compact thermal networks able to predict the dynamic junction temperature response under any arbitrary set of external cooling conditions have been represented by Christiaens et al. [52]. They have been successfully demonstrated for two types of polymer stud grid array (PSGA) packages, the standard PSGA and the thermally enhanced PSGA. Szekely developed the formulation of a thermal admittance matrix between different ports [53]. Every element in the matrix leads to a thermal network, and these networks appear coupled by voltage-controlled current sources. An equivalent network approach has been applied by Codecasa et al. to the analysis of the electrothermal effects of a metal oxide semiconductor field effect transistor (MOSFET) and bipolar junction transistor (BJT) devices [54]. They have shown how thermal effects, as they are seen at the electrical terminals, can be modeled through an equivalent purely electrical model obtained properly transforming the thermal impedance. The thermal network complexity reduction applied to a vertical power MOSFET device has been also investigated [55]. Few years later, Codecasa proposes passive compact models of dynamic thermal networks with many heat sources [56]. Recently, Li et al. 
used a nodal analysis method based on circuit analogy for simulating the responses of surface micromachined electrothermal micro-actuators and an outof-plane beamshaped electrothermal micro-actuator [42, 57]; a lumped approach that decomposes the static and dynamic performances of an U-shape micro-actuator into a series of thermal networks and a mechanical frame has been adopted by Lo et al. [58]; and Szabo and Szekely presented thermal networks, defined experimentally or analytically, of a quadratic transfer characteristics (QTC) element of which driving principle is the Seebeck effect [59].

Thermal modelling via electrical analogy is often applied to the thermal analysis of microelectronic and thermal microdevices, leading to complex thermal networks. Nevertheless, time set on thermal network simulations are set much shorter than on FEM simulations. Efforts to reduce the complexity of thermal networks are currently done.

\subsection{Model order reduction}

Simulation at the physical level including all geometrical details and physical interactions is not feasible, not even wished. Consequently, simulation is based on compact models at different levels of abstraction. Whereas numerical modeling can provide temperature distributions in the component with enough accuracy, the computing cost to simulate using meshing is extremely high, and it is of the most importance the development of compact dynamic thermal models. Automating the generation of low-dimensional systems of equations by means of mathematical techniques (e.g. Arnoldi, Balanced Truncation Approximation, $\lambda$-finder, and Guyan algorithms) has been widely studied $[60,61,62,63]$. Automatic model order reduction aims 
at providing reduced models only with minimal intervention by the designer. The goal is to provide a software based on a spatial discretization of the partial differential equations which is capable to return ordinary differential equations with a far lower number of state variables than the previous discretized system without sacrificing too much accuracy. These ordinary differential equations can then be simulated in acceptable time [60, 61]. Palacin et al. focus their attention to the development of compact thermal models from the analysis of the thermal impedance transients obtained from physical simulation (finite element model) [38]. Dynamic compact thermal models have been obtained for an ultrathin chip stacking technology where several chips can dissipate heat simultaneously. A multiport dynamic model has been obtained and the model has been implemented as a thermal impedance matrix. Different techniques for multiexponential signal analysis have been reviewed and compared (Jansson deconvolution, semiparametric exponential series method, constrained and free nonlinear least squares models).

Different methods searching modelling thermal problems have been reviewed. One way to model them is by using electrical analogy. Thermal network approach can provide rapid simulation (the model scale is much smaller than the FE model) with fairly high accuracy $[42,57]$. However, electrical analogy is not currently developed for beam geometries, commonly found on thermal micro-actuators, and having heat transfers through and around length. Next section deals with this thermal analysis via electrical analogy. 


\section{The electrical model}

Properties of electrothermal micro-actuators strongly depend on its geometric structure and material properties as well as applied voltage or current. It is therefore important to model the electrothermal micro-actuator so that its design may be improved or optimized. Thermal networks seem sufficiently accurate and powerful tool for simulating real thermal systems and microsystems. 3D heat transfers on thermal micro-actuators take place through and around long structures. This kind of geometries and thermal transfers has not been yet modelled using electrical analogy. We propose a new model applied to long structures. It is important to note that this study has been developed thinking particularly in thermal micro-actuators, but it can be applied to other thermal microsystems with the same beam geometries, such as microheaters. Table 2 summarizes equivalences between thermal and electrical systems.

Table 2: Thermal and electrical analogy

\begin{tabular}{ll}
\hline Thermal system & Equivalent electrical system \\
\hline Heat flux $Q$ & Current $i$ \\
Temperature difference $\Delta T$ & Voltage difference $\Delta V$ \\
\hline
\end{tabular}

This section is composed as follow: firstly, we analyse analytically the thermal behavior of the beam; secondly, the thermal equation is solved; thirdly, we propose the dynamic thermal network; and fourthly, the static thermal network is presented. 


\subsection{Dynamic thermal analysis}

In all the configurations of thermal micro-actuators (multimorph, Vshaped and U-shaped), heat transfers involve a combination of conduction and convection effects along structures. These structures and their thermal behavior can be generalized as the beam shown in Fig. 1(a): a heat flux $Q(x, t)$ through the beam causes a gradient of temperature between the two lateral faces at a temperatures $T_{1}$ and $T_{2}$, respectively; and a heat convection $Q_{h}$ takes place from all other surfaces in contact with the external fluid whose temperature is $T_{\text {ext }}$.

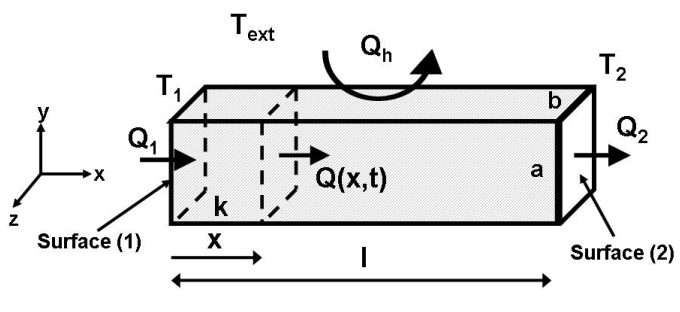

(a)

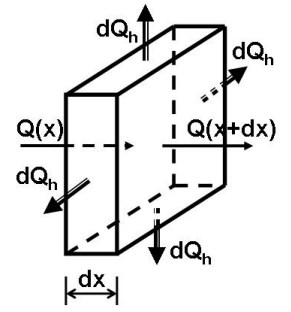

(b)

Figure 1: Structure analysed: (a) thermal problem, (b) elementary volume. hermal problem is reduced to a one dimensional problem.

Heat balance for the elementary volume in Fig. 1(b) establishes that difference between the incoming heat flux $Q(x)$ and the outgoing heat flux 
$Q(x+d x)$ is the sum of the convection heat flux and the stored heat flux which induces a variation of temperature:

$$
\frac{\partial Q(x)}{\partial x} d x=Q(x+d x)-Q(x)=-h P d x\left(T(x)-T_{e x t}\right)+\rho C_{p} S d x \frac{d T}{d t},
$$

${ }_{228}$ where $P=2 a+2 b$ is the lateral perimeter.

The conduction heat flux $Q(x)$ through the element is:

$$
Q(x)=-k S \frac{\partial T}{\partial x},
$$

where $S=a b$ is the lateral section.

Introducing (2) in (1), the evolution of the temperature $T=T(x, t)$ in the beam satisfies:

$$
\begin{array}{r}
\alpha \frac{\partial^{2} T}{\partial x^{2}}-\sigma\left(T-T_{e x t}\right)=\frac{\partial T}{\partial t} \\
\text { where } \quad \alpha=\frac{k}{\rho C_{p}} \quad \text { and } \quad \sigma=\frac{\rho C_{p} S}{h P} .
\end{array}
$$

Moreover, we are considering the following boundary and initial conditions:

$$
\begin{aligned}
Q_{1}=Q(0, t) & =-k S \frac{\partial T}{\partial x}(0, t) \\
Q_{2}=Q(l, t) & =-k S \frac{\partial T}{\partial x}(l, t) \\
T(x, 0) & =T_{e x t}
\end{aligned}
$$

Equations (3) to (6) define the thermal behavior of the beam.

\subsection{Resolution of the thermal equation}

The resolution of the thermal problem is done using Laplace transform, where $s$ is the parameter of the Laplace transform. Applying Laplace transform to $T(x, t)$, such as $\theta=\theta(x, s)=\operatorname{Laplace}[T(x, t)]$ and $\theta_{\text {ext }}=$ Laplace $\left[T_{\text {ext }}\right]=$ 
$\frac{T_{e x t}}{s}$, equation (3) can be written as:

$$
\begin{aligned}
& \frac{1}{q^{2}} \frac{d^{2} \theta}{d x^{2}}-\theta=-\theta_{\text {ext }} \\
& \text { where } \quad q^{2}=\frac{s+\sigma}{\alpha} .
\end{aligned}
$$

In the same way, the Laplace transform is applied to the boundary conditions (4) and (5):

$$
\begin{aligned}
& \phi_{1}=\text { Laplace }\left[Q_{1}\right]=-k S \frac{d \theta}{d x}(0, s) \\
& \phi_{2}=\text { Laplace }\left[Q_{2}\right]=-k S \frac{d \theta}{d x}(l, s)
\end{aligned}
$$

The solution of (7) considering (9) and (10) is thus:

$$
\theta(x, s)=\frac{\phi_{1} \cosh (q l)-\phi_{2}}{k S q \cdot \sinh (q l)} \cosh (q x)-\frac{\phi_{1}}{k S q} \sinh (q x)+\theta_{\text {ext }}
$$

where cosh and sinh are the hyperbolic cosine and the hyperbolic sine, respectively.

This non-linear equation represents the Laplace transform of the unidirectional temperature distribution $T(x, t)$ of any beam.

\subsection{Dynamic thermal model}

To propose the thermal network of the beam, heat flux $\phi_{1}$ and $\phi_{2}$ are first calculate as a function of $\theta_{1}=\theta(0, s)$ and $\theta_{2}=\theta(l, s)$ :

$$
\begin{aligned}
& \phi_{1}=-\frac{\theta_{\text {ext }}-\theta_{1}}{Z_{2}(q)}+\frac{\theta_{1}-\theta_{2}}{Z_{1}(q)} \\
& \phi_{2}=-\frac{\theta_{2}-\theta_{\text {ext }}}{Z_{2}(q)}+\frac{\theta_{1}-\theta_{2}}{Z_{1}(q)}
\end{aligned}
$$


where the thermal impedances $Z_{1}$ and $Z_{2}$ are:

$$
\begin{aligned}
& Z_{1}=\frac{\sinh (q l)}{k S q} \\
& Z_{2}=\frac{\sinh (q l)}{k S q(\cosh (q l)-1)}
\end{aligned}
$$

To be able to deduce the equivalent electrical model, thermal impedances $Z_{1 l i n}$ and $Z_{2 l i n}$ will be found using the infinite series expansion when $(q l) \rightarrow 0$. Based on (8), this limit tends to the consideration of only the slower and predominant dynamics $(s \rightarrow 0)$. Considering this limit, equations(14) and (15) tend to:

$$
\begin{aligned}
& Z_{1 \text { lin }}=R_{c 0} \\
& Z_{2 l i n}=\frac{1}{\frac{1}{R_{v 0}}+s C_{t h 0}}
\end{aligned}
$$

where the conduction thermal resistance $R_{c 0}$, the convection thermal resistance $R_{v 0}$ and the thermal capacitor $C_{t h 0}$ are respectively:

$$
\begin{aligned}
R_{c 0} & =\frac{l}{k S} \\
R_{v 0} & =\frac{2}{h P l} \\
C_{t h 0} & =\frac{\rho C_{p} S l}{2}
\end{aligned}
$$

Inserting (16) and (17) in (12) and (13), and applying the inverse Laplace transform, heat flux are:

$$
\begin{aligned}
& Q_{1}=-\frac{T_{e x t}-T_{1}}{R_{v 0}}+C_{t h 0} \frac{d T_{1}}{d t}+\frac{T_{1}-T_{2}}{R_{c 0}} \\
& Q_{2}=-\frac{T_{2}-T_{e x t}}{R_{v 0}}-C_{t h 0} \frac{d T_{2}}{d t}+\frac{T_{1}-T_{2}}{R_{c 0}}
\end{aligned}
$$

The dynamic thermal network of the beam described in Fig. 1 can be 238 constructed as schematized in Fig. 2. 


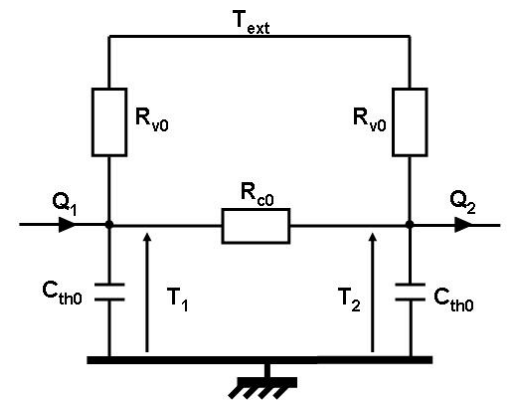

Figure 2: Dynamic thermal network of the beam.

\subsection{Static thermal network}

Here, we show that the static equivalent electrical model can be obtained without linearization, contrary to the dynamic model previously described.

Static impedances can be found using the general analytical study above. Impedances $Z_{1}$ and $Z_{2}$ in (14) and (15) respectively, depends on $q$, defined in (8). In the static case $s=0$, static thermal impedances, $Z_{1 s t}$ and $Z_{2 s t}$ are:

$$
\begin{aligned}
Z_{1 s t} & =\frac{L \sinh (l / L)}{k S} \\
Z_{2 s t} & =\frac{L \sinh (l / L)}{k S(1-\cosh (l / L))} \\
\text { where } \quad L^{2} & =\frac{k S}{h P}
\end{aligned}
$$

Both expressions, (23) and (24) are analytical expressions (not linearized) of static thermal impedances of the beam in Fig. 1. Using them in (12) and (13), analytical expressions of the static heat flux at $x=0$ and $x=l$ are:

$$
\begin{aligned}
Q_{1 s t} & =-\frac{k S}{L \sinh (l / L)}\left[\left(T_{e x t}-T_{1 s t}\right)(\cosh (l / L)-1)+\left(T_{2 s t}-T_{1 s t}\right)\right] \\
Q_{2 s t} & =-\frac{k S}{L \sinh (l / L)}\left[\left(T_{2 s t}-T_{e x t}\right)(\cosh (l / L)-1)+\left(T_{2 s t}-T_{1 s t}\right)\right]
\end{aligned}
$$


Static thermal network is thus deduced as shown in Fig. 3. This model considers only conduction and convection heat transfers represented respectively by thermal resistances $Z_{1 s t}$ and $Z_{2 s t}$.

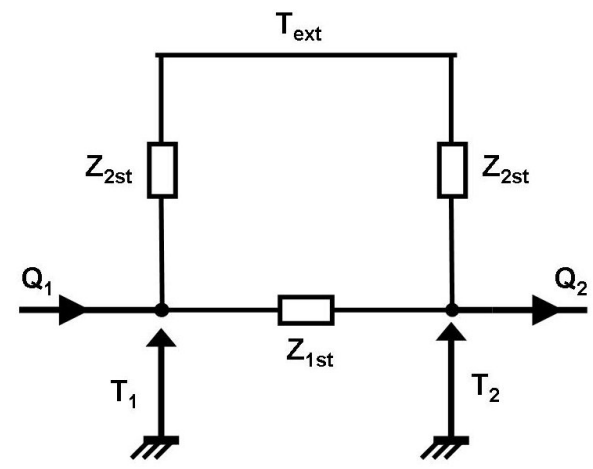

Figure 3: Static thermal network of the beam.

Next section deals with the validity of thermal networks obtained from linearized thermal impedances.

\section{Validity of thermal networks}

Thermal impedances $Z_{1 l i n}$ and $Z_{2 l i n}$, and consequently thermal networks, have a validity condition because they have been obtained thanks to the linearization of impedances $Z_{1}$ and $Z_{2}$. This section deals with the validity of the impedances, the validity of thermal networks, and the scaling effect of the validity criteria.

\subsection{Validity of thermal impedances}

Impedances $Z_{1 l i n}$ and $Z_{2 l i n}$ are only valid when they are near to the analytical impedances $Z_{1}$ and $Z_{2}$. Applying a second order Taylor development 
to impedances in (14) and (15), they can be written as:

$$
\begin{aligned}
& Z_{1} \approx \frac{l}{k S}\left(1+\frac{(q l)^{2}}{6}\right)=Z_{1 \operatorname{lin}}\left(1+\frac{(q l)^{2}}{6}\right) \\
& Z_{2} \approx \frac{2}{k S l q^{2}}\left(1+\frac{(q l)^{2}}{12}\right)=Z_{2 l i n}\left(1+\frac{(q l)^{2}}{12}\right)
\end{aligned}
$$

The dynamic thermal network model is valid if at least:

$$
\left|\frac{(q l)^{2}}{6}\right| \ll 1
$$

In case of an sinusoidal input with an angular frequency $\omega(s=j \omega)$, and remembering (8), condition (29) is expressed by:

$$
\omega^{2} \ll \omega_{v}^{2}
$$

where:

$$
\omega_{v}^{2}=\frac{36 \alpha^{2}}{l^{4}}-\sigma^{2}=\frac{36}{l^{4}}\left(\frac{k}{\rho C_{p}}\right)^{2}-\left(\frac{h P}{\rho C_{p} S}\right)^{2}
$$

254 Linear thermal impedances are thus valid only when angular frequency $\omega$ is 255 lower than the characteristic frequency $\omega_{v}$, which includes all the parameters

256 of the beam.

\subsection{Validity of the dynamic thermal network}

The objective of this section is to compare the frequency $\omega_{v}$ with the poles of the dynamic thermal network. Based on the dynamic thermal network in Fig. 2, two poles $s_{1}$ and $s_{2}$ can be calculated:

$$
\begin{aligned}
& s_{1}=-\frac{1}{R_{v 0} C_{t h 0}} \\
& s_{2}=-\frac{R_{c 0}+2 R_{v 0}}{R_{c 0} R_{v 0} C_{t h 0}}
\end{aligned}
$$


The poles are the representation of the characteristic frequencies of the dynamic system, thus considering (30), poles $s_{1}$ and $s_{2}$ have to respect:

$$
\begin{aligned}
\left|s_{1}\right| & \ll \omega_{v}^{2}, \\
\text { and }\left|s_{2}\right| & \ll \omega_{v}^{2} .
\end{aligned}
$$

Decomposing the terms, conditions (34) and (35) can be defined as a unique condition, which defines the validity of the dynamic thermal network:

$$
\begin{aligned}
\nu & \ll 1 \\
\text { where } \quad \nu & =\frac{h P l^{2}}{(\sqrt{14}-2) k S}
\end{aligned}
$$

Criterion $\nu$ establishes the validity zone of the dynamic thermal network.

\subsection{Validity of the static results of thermal networks}

The network defined in Fig. 3 which uses $Z_{1 s t}$ and $Z_{2 s t}$ is always valid whatever the geometry is. This section deals with the definition of validity domain of the static results obtained with the dynamical network defined in Fig. 2. In this case, the condition of validity (30), knowing that $\omega=0$, becomes:

$$
\begin{aligned}
\delta & \ll 1 \\
\text { where } \quad \delta & =\frac{h P l^{2}}{6 k S}
\end{aligned}
$$

Criterion $\delta$ establish the validity zone for the static results of the network defined in Fig. 2. 


\subsection{Scaling effect on validity criteria}

This section is dedicated to the study of the scale effect on this criterion. If $d$ is the characteristic size of the beam, criteria $\nu$ and $\delta$ are:

$$
\delta \sim \nu \sim \frac{h d^{3}}{k l^{2}} \sim \frac{h}{k} d
$$

Thermal conductivity $k$ is an intrinsic property of the material and is thus independent of scaling effects, so:

$$
\delta \sim \nu \sim h d
$$

In the macroworld, convection coefficient $h$ is considered constant and independent of the scaling effects: criteria $\nu$ and $\delta$ are proportional to the size $d$. Consequently, the validity of thermal networks tends to be improved when size reduces. In the microworld, convection coefficient $h$ is considered inversely proportional to size $d\left(h \sim d^{-1}\right)$, criteria $\nu$ and $\delta$ are, consequently, independents of size $d$. This study of the scale effect shows the relevance of our models in the microscale.

In the next section, we compare thermal networks to analytical results and FEM simulations.

\section{Validation of thermal networks}

The objective of this section is to compare our models with analytical and FE models for different values of the criteria $\delta$ and $\nu$.

We consider a silicon beam whose physical parameters are defined in Table 3. The external temperature $T_{\text {ext }}$ is considered constant at $293 \mathrm{~K}$. The geometrical parameters $a$ and $b$ are constant in all the calculations:

$$
a=200 \mu \mathrm{m} \quad \text { and } \quad b=500 \mu \mathrm{m}
$$


The length $l$ is modified in order to obtain several criteria $\delta$ and $\nu$. Moreover, to take into account a large diversity of applications, tests are proposed in water and air. FEM simulations are done with COMSOL Multiphysics ${ }^{\text {TM }}$ 3.2. The meshed model consists of triangular elements on the surface and tetrahedral elements in the volume. The automatic meshing available in COMSOL Multiphysics has been used to define the meshing. Depending on the geometry of the beam, the mesh includes 5700 to 8800 elements which represent between 1300 and 2100 nodes. The thermal network is simulated using Simulink ${ }^{\mathrm{TM}}$ and Matlab $^{\mathrm{TM}}$.

Table 3: Physical properties of tested beams.

\begin{tabular}{lrl}
\hline Parameter & Value & Unit \\
\hline$k$ & 148 & $\mathrm{~W} \cdot \mathrm{m}^{-1} \cdot \mathrm{K}^{-1}[64]$ \\
$h_{\text {air }}$ & 924 & $\mathrm{~W} \cdot \mathrm{m}^{-2} \cdot \mathrm{K}^{-1}[42]$ \\
$h_{\text {water }}$ & 9240 & $\mathrm{~W} \cdot \mathrm{m}^{-2} \cdot \mathrm{K}^{-1}[42,65]$ \\
$\rho$ & 2330 & $\mathrm{~kg} \cdot \mathrm{m}^{-3}[64]$ \\
$C_{p}$ & 705 & $\mathrm{~J} \cdot \mathrm{kg}^{-1} \cdot \mathrm{K}^{-1}[64]$ \\
\hline
\end{tabular}




\subsection{Validation of the dynamic thermal network}

In order to validate the thermal network, the heat flux applied $Q_{1}(t)=$ $Q(0, t)$ and $Q_{2}(t)=Q(l, t)$ are:

$$
\begin{aligned}
& Q_{1}(t)= \begin{cases}0 & \text { for } t<0 \\
0.3 \mathrm{~W} & \text { for } t \geq 0\end{cases} \\
& Q_{2}(t)= \begin{cases}0 & \text { for } t<0 \\
-0.1 \mathrm{~W} & \text { for } t \geq 0\end{cases}
\end{aligned}
$$

The temperatures $T_{1}(t)=T(0, t)$ and $T_{2}(t)=T(l, t)$ have been calculated in air and water using FEM calculations, and compared to the equations obtained from the thermal network in Fig. 2. Table 4 shows, in air and water, the criterion $\nu$ for two different lengths $l$, leading to two different geometries, I and II.

Table 4: Geometries and their validation parameter in air and water.

\begin{tabular}{cccc}
\hline Geometry & Length $(\mu \mathrm{m})$ & Criterion $\nu$ in air & Criterion $\nu$ in water \\
\hline I & $l=500$ & 0.01 & 0.1 \\
II & $l=3000$ & 0.45 & 4.5 \\
\hline
\end{tabular}

Figures 4(a) and 4(c) present the obtained results with geometry I respectively in both media, air and water, while figures 4(b) and 4(d) illustrate the results obtained with geometry II respectively in air and water. As the criterion $\nu$ in Fig. 4(a) is better than the criterion $\nu$ in Fig. 4(d), thermal networks curves are closer to FEM curves than in Fig. 4(a).

These experiments show a good reliability of our model compared to the FEM calculations and show the relevance of the dynamic criteria $\nu$. 


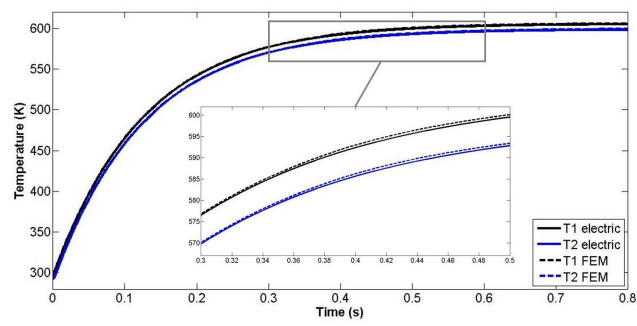

(a) Geometry I in air $(\nu=0.01)$.

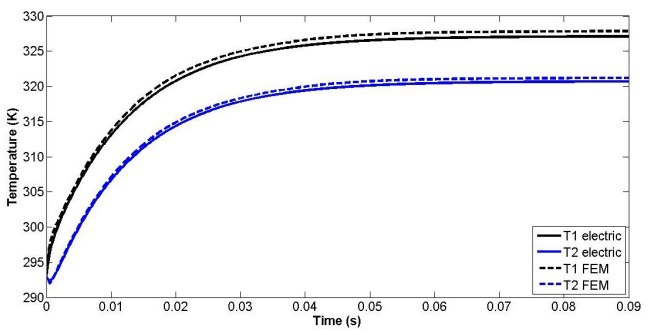

(c) Geometry I in water $(\nu=0.1)$.

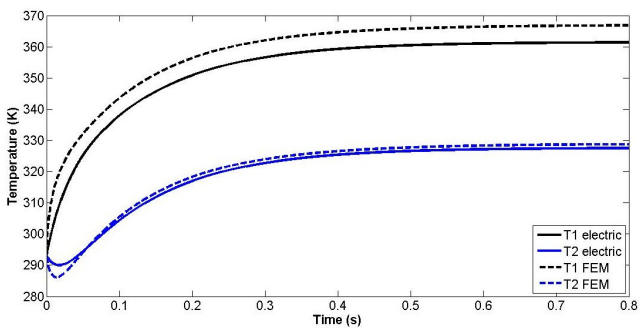

(b) Geometry II in air $(\nu=0.45)$.

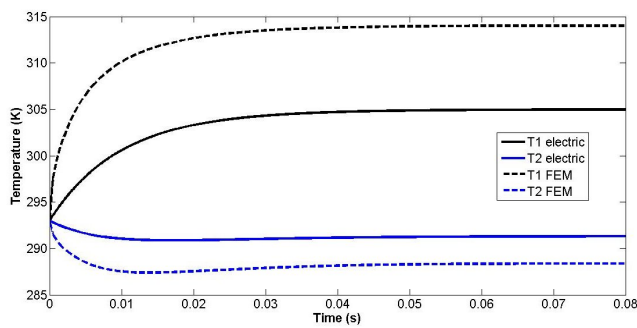

(d) Geometry II in water $(\nu=4.5)$.

Figure 4: $T_{1}$ and $T_{2}$ for different geometries.

\subsection{Dynamic behaviour}

The time dependent behaviour is investigated in the angular frequency $(\omega)$ domain. A heat flux $Q_{1}(t)=\sin (\omega t)$ is imposed, keeping $Q_{2}(t)=0$. Influence of this last, can be deduced by symmetry. The evolution of the temperatures $T_{1}(t)$ and $T_{2}(t)$ obtained by FEM simulations and the thermal network in Fig. 2 for geometry II in air $(\nu=0.45)$, are plotted in Fig. 5. These curves confirm the good reliability of the proposed model. 


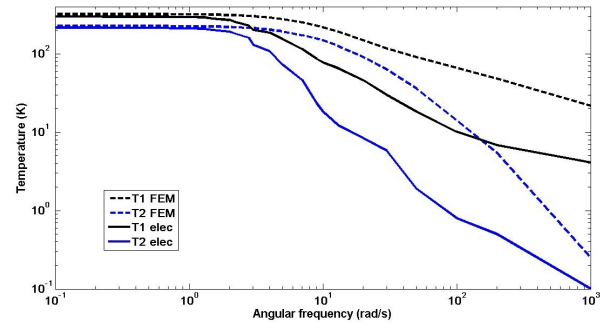

Figure 5: Dynamic behaviour for geometry II in air.

\subsection{Validation of the static thermal network}

In order to validate the static thermal network, constant temperatures $T_{1 s t}=393 \mathrm{~K}$ and $T_{2 s t}=293 \mathrm{~K}$ are imposed, and heat flux $Q_{1 s t}$ and $Q_{2 s t}$, for different geometries in water and air, have been calculated using three different models: the static network (SN) in Fig. 3, the static results of the dynamic network (DN) in Fig. 2, and FEM simulations.

The comparison of the values for several cases is presented in Table 5 . Let us note that the criterion $\delta$ obtained in (39) defines the validity of the static results of the dynamic network.

First, these results show a good reliability of the static network (SN) compared to FEM simulations whatever the criterion $\delta$ is. In fact, the definition of the impedances $Z_{1 s t}$ and $Z_{2 s t}$ has been done without linearization. The good results obtained comparing either analytical or FEM calculation with the dynamic or static thermal networks enable us to validate them.

Concerning the static results of the dynamic network (DN, as shown in Fig. 6), the percentage of error is growing when the criterion $\delta$ is approaching to 1 . In conclusion, validity criteria $\delta$ must also be considered relevant. 
Table 5: Comparison of heat flux $Q_{1}$ and $Q_{2}$ in air and water.

\begin{tabular}{llllllll}
\hline \multirow{2}{*}{$\begin{array}{l}\text { Length } l \\
(\mu \mathrm{m})\end{array}$} & Model & \multicolumn{3}{c}{ Air } & \multicolumn{4}{c}{ Water } \\
\cline { 3 - 8 } & & $\delta$ & $Q_{1 s t}$ & $Q_{2 s t}$ & $\delta$ & $Q_{1 s t}$ & $Q_{2 s t}$ \\
& & & $(\mathrm{~W})$ & $(\mathrm{W})$ & & $(\mathrm{W})$ & $(\mathrm{W})$ \\
\hline \multirow{2}{*}{500} & $\mathrm{SN}$ & & 2.98 & 2.95 & & 3.17 & 2.85 \\
& FEM & & 2.98 & 2.95 & & 3.17 & 2.85 \\
& $\mathrm{DN}$ & 0.004 & 2.99 & 2.93 & 0.04 & 3.28 & 2.64 \\
\hline \multirow{2}{*}{1000} & $\mathrm{SN}$ & & 1.52 & 1.46 & & 1.89 & 1.28 \\
& FEM & & 1.52 & 1.46 & & 1.88 & 1.28 \\
& $\mathrm{DN}$ & 0.01 & 1.54 & 1.42 & 0.1 & 2.13 & 0.83 \\
\hline \multirow{2}{*}{1500} & SN & & 1.05 & 0.96 & & 1.56 & 0.72 \\
& FEM & & 1.05 & 0.95 & & 1.55 & 0.73 \\
& DN & 0.03 & 1.08 & 0.89 & 0.3 & 1.96 & 0.02 \\
\hline
\end{tabular}

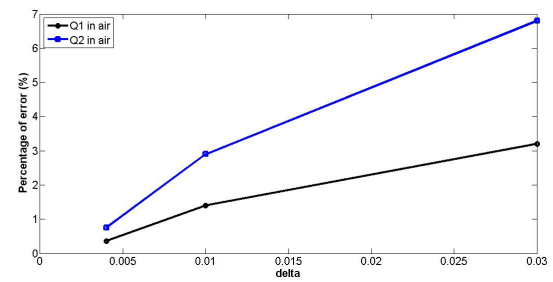

(a) In air.

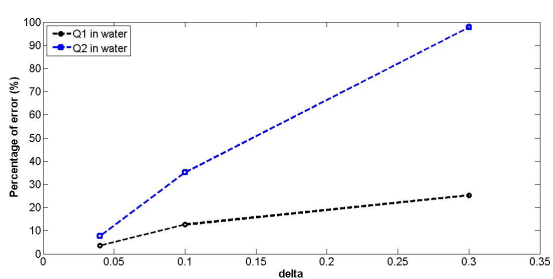

(b) In water.

Figure 6: Percentage of error between the static results of dynamic network and the static network, with respect to $\delta$. 


\section{Conclusion}

Thermal micro-actuators are one of the most promising methods to induce movements in micromechatronics. The different configurations (multimorph, U-shaped and V-shaped) have a common geometry: long structures. As the optimization and control of their heat transfers are a crucial part to perform micro-actuators, we propose to model these systems using electrical analogy. To our knowledge, until now, only classical thermal networks have been developed for thermal systems like walls. In this paper, we develop the dynamic and static equivalent electrical models of long structures. The validity of both thermal networks, dynamic and static, have been also established. Finite elements and analytical results have been compared with results obtained using our thermal networks, in order to validate them. In all the studied cases, they appear sufficiently accurate. Moreover, as the thermal parameters of the proposed thermal networks are defined by the physical properties of the studied structures, constraints and dependences of these properties in relation to other properties could be directly addressed by the thermal networks. The compilation of elementary thermal networks proposed in this paper has been done in order to model a complex thermal microdevice [66].

\section{Acknowledgment}

This work is supported by the CONACYT Mexican National Council for Science and Technology, and the French research project PRONOMIA ANR No. 05-BLAN-0325-01. 


\section{References}

[1] K.-M. Liao, C.-C. Chueh, R. Chen, A novel electro-thermally driven bi-directional microactuator, in: Proc. of International Symposium on Micromechatronics an Human Science, 2002, pp. 267-274.

[2] S. H. Lee, K.-C. Lee, S. S. Lee, H.-S. Oh, Fabrication of an electrothermally actuated electrostatic microgripper, in: Proc. of Transducers, 2003, pp. 552-555.

[3] P. Robert, D. Saias, C. Billard, S. Boret, N. Sillon, C. Maeder-Pachurka, P. L. Charvet, G. Bouche, P. Ancey, P. Berruyer, Integrated rf-mems switch based on a combination of thermal and electrostatic actuation, in: Proceedings of Transducers, Vol. 2, 2003, pp. 1714-1717.

[4] E. Bassous, H. H. Taub, L. Kuhn, Ink jet printing nozzle arrays etched in silicon., Applied Physical Letters 31 (1977) 135.

[5] M. Tokeshi, J. Yamaguchi, A. Hattori, T. Kitamori, Thermal lens micro optical systems, Analytical Chemistry 77 (2) (2005) 626-630.

[6] S.-Y. Lee, H.-W. Tung, W.-C. Chen, W. Fang, Thermal actuated solid tunable lens, IEEE Photonics Technology Letters 18 (21) (2006) 21912193.

[7] A. Pantazi et al., A servomechanism for a micro-electro-mechanicalsystem-based scanning-probe data storage device, Nanotechnology 15 (10) (2004) S612-S621. 
[8] C. G. Slough, A. Hammiche, M. Reading, H. M. Pollock, Photo thermal micro-spectroscopy - a new method for infrared analysis of materials, Journal of ASTM International 2 (10) (2005) pp. 6.

[9] B. H. Weiller, P. D. Fuqua, J. V. Osborn, Fabrication, characterization, and thermal failure analysis of a micro hot plate chemical sensor substrate, Journal of the Electrochemical Society 151 (3) (2004) H59-H65.

[10] N.-F. Chiu, T.-C. Hsiao, L. C-W., Low power consumption design of micro-machined thermal sensor for portable spirometer, Tamkang Journal of Science and Engineering 8 (3) (2005) 225-230.

[11] J. Han, Z. Tan, K. Sato, M. Shikida, Thermal characterization of micro heater arrays on a polyimide film substrate for fingerprint sensing applications, Journal of Micromechanics and Microengineering 15 (2) (2005) 282-289.

[12] T. H. Kim, S. J. Kim, Development of a micro-thermal flow sensor with thin-film thermocouples, Journal of Micromechanics and Microengineering 16 (11) (2006) 2502-2508.

[13] Z. Tan et al., Experimental and theoretical study of an on-wall in-tube flexible thermal sensor, Jounal of Micromechanics and Microengineering 17 (4) (2007) 679-686.

[14] A. Jain, H. Qu, S. Todd, H. Xie, A thermal bimorph micromirror with large bi-directional and vertical actuation, Sensors and Actuators A 122 (2005) 9-15. 
[15] M. Boutchich, T. J. Mamtora, G. J. McShane, I. Haneef, D. F. Moore, J. A. Williams, Force measurements on u-shaped electrothermal microactuators: applications to packaging., Proc. of the IMechE Part C: Journal of Mechanical Engineering Science 222 (2008) 87-96.

[16] H.-Y. Chan, W. J. Li, A thermally actuated polymer micro robotic gripper for manipulation of biological cells, in: Proc. of IEEE ICRA, Taiwan, 2003, pp. 288-293.

[17] R. Hickey, D. Sameoto, T. Hubbard, M. Kujath, Time and frequency response of two-arm micromachined thermal actuators, Journal of Micromechanics and Microengineering 13 (2002) 40-46.

[18] L. Li, D. Uttamchandani, Modified asymmetric micro-electrothermal actuator: analysis and experimentation, Journal of Micromechanics and Microengineering 14 (2004) 1734-1741.

[19] N.-T. Nguyen, S.-S. Ho, C. L.-N. Low, A polymeric microgripper with integrated thermal actuators, Journal of Micromechanics and Microengineering 14 (2004) 969-974.

[20] D. Yan, A. Khajepour, R. Mansour, Design and modeling of a mems bidirectional vertical thermal actuator, Journal of Micromechanics and Microengineering 14 (2004) 841-850.

[21] R. M. Hickey, Thermal actuator, Tech. rep., uS Patent (2006).

[22] M. J. Sinclair, A high force low area mems thermal actuator, in: Proc of ITHERM, Vol. 1, 2000, pp. 127-132. 
[23] A. Unamuno, J. Yao, D. Uttamchandani, Alignment and fixing of fiber optics based on electrothermal mems actuators, IEEE Photonics Technology Letters 17 (4) (2005) 816-818.

[24] Y. Lai, E. V. Bordatchev, S. K. Nikumb, W. Hsu, Performance characterization of in-plane electro-thermally driven linear microactuators, Journal of Intelligent Materials Systems and Structures 17 (2006) 919929.

[25] M. J. F. Zeman, E. V. Bordatchev, G. K. Knopf, Design, kinematic modeling and performance testing of an electro-thermally driven microgripper for micromanipulation applications, Journal of Micromechanics and Microengineering 16 (2006) 1540-1549.

[26] N. Chronis, L. P. Lee, Electrothermally activated su-8 microgripper for single cell manipulation in solution, Journal of Microelectromechanical Systems 14 (4) (2005) 857-863.

[27] M. Mayyas et al., Design tradeoffs for electrothermal microgrippers, in: Proc. of IEEE ICRA, 2007, pp. 907-912.

[28] X. J. Hu, A. Jain, K. E. Goodson, Investigation of the natural convection boundary condition in microfabricated structures, International Journal of Thermal Sciences 47 (2008) 820-824.

[29] K. L. Zhang, S. K. Chou, S. S. Ang, Fabrication, modeling and testing of a thin film au/ti microheater, International Journal of Thermal Sciences 46 (2007) 580-588. 
[30] D. DeVoe, Thermal issues in mems and microscale systems, Transactions on Components and Packaging Technologies 25 (4) (2003) 576-583.

[31] A. Pessiot-Bonvilain, étude et réalisation d'un microrobot à pattes : structure mécanique et micro-actionnement, Ph.D. thesis, Université de Franche-Comté (2002).

[32] B. López-Walle, M. Gauthier, N. Chaillet, Principle of a submerged freeze gripper for microassembly, IEEE Transactions on Robotics 24 (4) (2008) 897-902.

[33] L.-N. Tsai, G.-R. Shen, Y.-T. Cheng, W. Hsu, Performance improvement of an electrothermal microactuator fabricated using ni-diamond nanocomposite, Journal of Microelectromechanical Systems 15 (1) (2006) 149-158.

[34] H. Janocha (Ed.), Adaptatronics and smart structures, Springer, 1999.

[35] J. Peirs, Design of micromechatronic systems: scale laws, technologies, and medical applications, Ph.D. thesis, Catholic University of Leuven (2001).

[36] T. Moulton, G. K. Ananthasuresh, Micromechanical devices embedded eletro-thermal-compliant actuation, Sensors and Actuators A 90 (2001) $38-48$.

[37] D. O. Popa, B. H. Kang, J. T. Wen, Dynamic modeling and input shaping of thermal bimorph mems actuators, in: Proc. of IEEE ICRA, 2003, pp. 1470-1475. 
[38] J. Palacin, M. Salleras, J. Samitier, S. Marco, Dynamic compact thermal models with multiple power sources: application to and ultrathin chip stacking technology, IEEE Transactions on Advanced Packaging 28 (4) (2005) 694-703.

[39] G. K. Lau, J. F. L. Goosen, F. van Keulen, T. Chu Duc, P. M. Sarro, Powerful polymeric thermal microactuator with embedded silicon microstructure, Applied Physics Letters 90 (2007) 214103.

[40] C. Elbuken, N. Topaloglu, P. M. Nieva, M. Yavuz, J. P. Huissoon, Modeling and analysis of a 2-dof bidirectional electro-thermal microactuator, Microsystem Technologies 15 (2009) 713-722.

[41] M. Mayyas, H. Stephanou, Electrothermoelastic modeling of mems gripper, Microsystem Technologies 15 (2009) 637-646.

[42] R. Li, Q.-A. Huang, W. Li, A nodal analysis model for the out-ofplane beamshape electrothermal microactuator, Microsystem Technologies 15 (2) (2009) 217-225.

[43] C.-T. Wu, W. Hsu, Design and fabrication of an electrothermal microactuator for multi-level conveying, Microsystem Technologies 12 (2006) $293-298$.

[44] W.-C. Tai, C.-T. Wu, C.-P. Hsu, W. Hsu, Tdesign and fabrication of a three-dimensional long-stretch micro drive by electroplating, in: Proc. of International Conference on MEMS, 2004, pp. 474-477.

[45] M. Shamshirsaz, M. Maroufi, M. B. Asgari, M. Gheisarieha, Influence of material stiffness and geometrical variations on the electro-thermally 
driven microactuator performance, Microsystem Technologies 15 (2009) 155-159.

[46] V. Paschkis, H. D. Baker, A method for determining unsteady-state heat transfer by means of an electrical analogy., Transactions ASME 64 (1942) 105-112.

[47] M. Jakob, Heat transfer, Vol. 1, McGraw-Hill, New York, 1949.

[48] J. P. Holman, Heat transfer, Mac Graw Hill, 1990.

[49] B. Borovic et al., Method for determining a dynamical state-space model for control of thermal mems devices, Journal of Microelectromechanical Systems 14 (5) (2005) 961-970.

[50] J. Zueco, A. Campo, Network model for the numerica simulation of transient radiative transfer process between the thick walls of enclosures, Applied Thermal Engineering 26 (2006) 673-679.

[51] C. Cristofari, G. Notton, A. Louche, Study of the thermal behaviour of a production unit of concrete structural components, Applied Thermal Engineering 24 (2004) 1087-1101.

[52] F. Christiaens, B. Vandevelde, E. Beyne, R. Mertens, J. Berghmans, A generic methodology for deriving compact dynamic thermal models, applied to psga package, IEEE Transactions on Components, Packaging, and Manufacturing Technology-Part A 21 (4) (1998) 565-576.

[53] V. Szekely, Identification of rc networks by deconvolution: chances and 
limits, IEEE Transactions on Circuits and Systems I, Fundamental Theory and Applications 45 (3) (1998) 244-258.

[54] L. Codecasa, D. D'Amore, P. Maffezzoni, Modeling the thermal response od semiconductor devices through equivalent electrical networks, IEEE Transactions on Circuits and Systems-I: Fundamental Theory and Applications 49 (8) (2002) 1187-1197.

[55] L. Codecasa, D. D'Amore, P. Maffezzoni, Compact modeling of electrical devices for electrothermal analysis, IEEE Transactions on Circuits and Systems-I: Fundamental Theory and Applications 50 (4) (2003) 465-476.

[56] L. Codecasa, Compact models of dynamic thermal networks with many heat sources, IEEE Transactions on Components and Packaging Technologies 30 (4) (2007) 653-659.

[57] R.-G. Li, Q.-A. Huang, W.-H. Li, A nodal analysis method for simulating the behavior of electrothermal microactuators, Microsystem Technologies 14 (2007) 119-129.

[58] C.-C. Lo, M.-J. Lin, C.-L. Hwan, Modeling and analysis of electrothermal microactuators, Journal of the Chinese Institute of Engineers 32 (2009) 351-360.

[59] P. G. Szabo, V. Szekely, Characterization and modeling of electrothermal mems structures, Microsystem Technologies 15 (2009) 1293-1301.

[60] T. Bechtold, E. B. Rudnyi, J. G. Korvink, Automatic generation of compact electro-thermal models for semiconductor devices, IEICE Transactions on Electronics E86 (C3) (2003) 459-465. 
[61] J. Lienemann, D. Billger, E. B. Rudnyi, A. Greiner, J. G. Korvink, Mems compact modeling meets model order reduction: examples of the application of arnoldi methods to microsystem devices, in: In Technical Proceedings of the 2004 NSTI Nanotechnology Conference and Trade Show, Nanotech 2004, Vol. 2, Massachusetts, USA, 2004, pp. 303-306.

[62] E. B. Rudny, J. Lienemann, A. Greiner, J. G. Korvink, mor4ansys: Generating compact models directly from ansys models, in: In Technical Proceedings of the 2004 NSTI Nanotechnology Conference and Trade Show, Nanotech 2004, Vol. 2, Massachusetts, USA, 2004, pp. 279-282.

[63] E. Kollar, V. Szekely, Reducing the possibility of subjective error in the determination of the structure-function-based effective thermal conductivity of boards, in: In Proc. of the IEEE THERMINIC Workshop, Nice, France, 2006.

[64] K. Ivanova et al., Thermally driven microgripper as a tool for micro assembly, Microelectronic Engineering 83 (4-9) (2006) 1393-1395.

[65] J. Abadie, N. Chaillet, C. Lexcellent, An integrated shape memory alloy micro-actuator controlled by the thermoelectric effect, Sensors and Actuators A 99 (2002) 297-303.

[66] B. López-Walle, M. Gauthier, N. Chaillet, Dynamic modelling for a submerged freeze microgripper using thermal networks, Journal of Micromechanics and Microengineering 20 (2) (2010) 025001 (10pp). 